\title{
Was wirklich wichtig ist
}

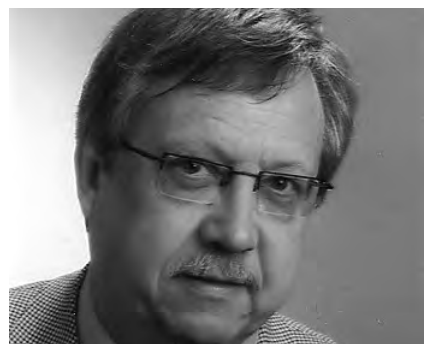

VON GEORG HORCHER Georg Horcher ist Leiter des Sozial- und Jugendamtes des Kreises Offenbach.

E-Mail

g.horcher@kreis-offenbach.de

\author{
Mit einer Festschrift anderer Art beschreiben Freunde \\ und Weggefährten den beruflichen Weg und das \\ Lebenswerk von Bernd Maelicke zu dessen 70. \\ Geburtstag. Sie verknüpfen darin die Entwicklungslinien \\ der beiden Handlungsfelder Strafvollzug und \\ Sozialwirtschaft, die beide von Bernd Maelicke \\ wesentlich beeinflusst und mitgestaltet wurden.
}

»Wertschöpfung « und »Wertschätzung « sind die verbindenden Begriffe erfolgreicher Praxis sowohl in der Resozialisierungsarbeit als auch erfolgreichen Managens in der Sozialwirtschaft. Beide Begriffe kennzeichnen auch das erfolgreiche Schaffen von Bernd Maelicke.

Im ersten Teil beschreibt Frieder Dünkel die Lebensleistung von Bernd Maelicke entlang der Entwicklung des Diskurses der letzten 35 Jahre um die Reform des Strafvollzugs und die Resozialisierung. Armin Wöhrle stellt die Innovationslinien des Sozialmanagements und die Rolle, die Bernd Maelicke dabei gespielt hat dar. Ergänzt werden diese beiden Beiträge durch einen Beitrag von Andreas Tietze, der in einfühlsamer Weise am Beispiel der Person des Jubilars, die ethischen und normativen Grundlagen erfolgreichen sozialen Unternehmertums beschreibt. Peter Zängl schließt den ersten Teil des Buches mit einem persönlichen, erfahrungsbezogenen Beitrag zur Leistung von Bernd Maelicke zur Seniorenpolitik und Seniorenwirtschaft ab.

Im zweiten Teil beschreiben eine Reihe von Weggefährten aus Wissenschaft, Ministerialbürokratie, der Justiz und der Sozialen Arbeit episodenhaft wie sie Bernd Maelicke kennen und schätzen gelernt haben. Sie geben damit Einblicke nicht nur in die scheinbar fast unbegrenzte Schaffenskraft von Bernd Maelicke und seine Person, sondern als
Zeitzeugen auch in die Entwicklung der Sozialen Arbeit und der Sozialwirtschaft.

In einem weiteren Beitrag würdigt Ursula Müller die Ehefrau von Bernd Maelicke, Hannelore Maelicke, die zunächst im Frauenstrafvollzug und dann als Gleichstellungsbeauftragte er-

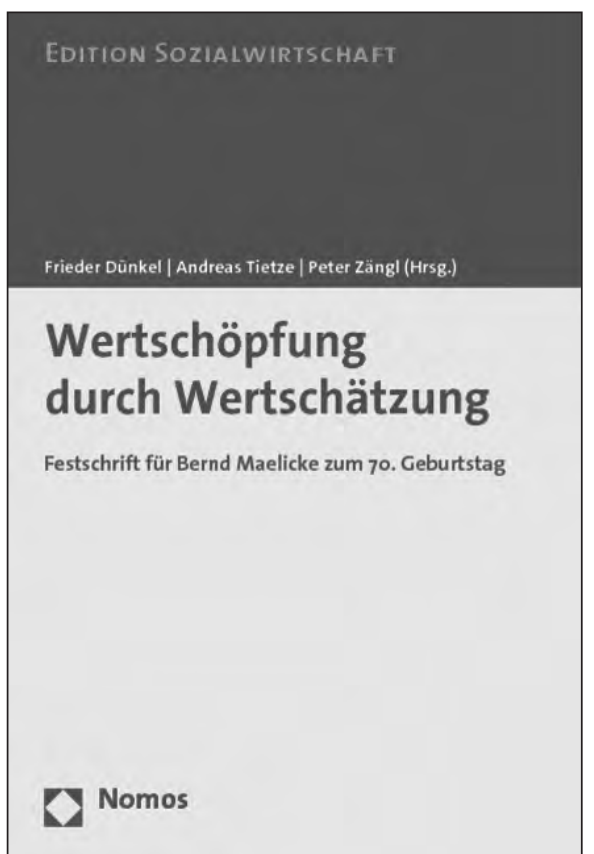

Frieder Dünkel, Andreas Tietze, Peter Zängl (Hg.): Wertschöpfung durch Wertschätzung. Festschrift für Bernd Maelicke zum 70. Geburtstag. Nomos Verlagsgesellschaft, Baden-Baden 2011. 191 Seiten. 29,- Euro. ISBN 978-3-8329-6534-1. 
folgreich eine eigenständige berufliche Karriere eingeschlagen und mit Sachverstand und Wertschätzung die Gleichstellung von Frauen und Männern vorangebracht hat.

Das Buch schließt mit einem Interview, das Daniela Friedrich mit Bernd Maelicke geführt hat, in dem er sein Leben selbstkritisch und humorvoll Revue passieren lässt und mit einem nach Erscheinungsjahren gegliederten, vollständigen Schriftenverzeichnis, das von 1975 bis 2011 insgesamt 210 Titel umfasst.

Das Buch beschreibt also nicht nur das Lebenswerk eines Mannes, der sowohl in der Strafvollzugs- als auch in der Sozialmanagement-Debatte in den letzten Jahren deutliche Spuren hinterlassen hat. Wer sich einen gerafften aber, inhaltlich fast vollständigen Überblick über die Reform des Strafvollzugs verschaffen und gleichzeitig den Einfluss von Bernd Maelicke auf diesen Gegenstandsbereich in Erfahrung bringen will, findet im Beitrag von Frieder Dünkel »Resozialisierung als Lebensthema. 5 Jahrzehnte Forschung und Praxisentwicklung « eine hervorragende Grundlage. Der Autor beschreibt die Grundlinien der Auseinandersetzung in zeitlicher Abfolge und die jeweilige Grundposition von Bernd Maelicke. Es wird schon in diesem Beitrag sichtbar, was später auch den Beitrag von Bernd Maelicke zur Entwicklung des Sozialmanagements prägt: systemisches Denken und Handeln. Der Beitrag stellt eindrücklich dar, wie Bernd Maelicke sich schon früh für die Zusammenfassung der Gerichtshilfe, der Bewährungshilfe und der Führungsaufsicht in einem einheitlichen Sozialen Dienst der Justiz einsetzte und Konzepte für ein Übergangsmanagement vom Strafvollzug in die Freiheit entwarf.

Für Maelicke war schon früh klar, dass erfolgreiche Resozialisierung den Aufbau und die Entwicklung tragfähiger Netzwerke bedarf. Ganzheitliche Organisationsstrukturen, Netzwerkarbeit und systemisches Handeln prägen auch die Entwicklung des Sozialmanagements und der Seniorenwirtschaft. Für den Bereich der Seniorenwirtschaft und Seniorenpolitik beschreibt Peter Zängl in einem kurzen, aber prägnanten Artikel, der auch anschaulich macht, wie es Bernd Maelicke gelang, seine theoretischen und normativen Anforderungen an ein innovatives Sozialmanagement in praktisches Handeln umzusetzen.

Der Beitrag von Armin Wöhrle »Innovation und Management in der Sozialwirtschaft - zur Verortung von Bernd Maelicke « befasst sich mit der Entwicklung der Sozialwirtschaft und macht die Rolle von Bernd Maelicke als einen der prägenden Innovatoren deutlich. Besonders lesenswert sein Interview mit dem Jubilar am Ende des Beitrags. In diesem Interview zieht Bernd Maelicke eine fachliche Bilanz seines zweiten Arbeitsschwerpunkts, dem Sozialmanagement und der Sozialwirtschaft.

Für beide Felder, das Sozialmanagement und die Resozialisierung und deren Weiterentwicklung, formuliert Bernd Maelicke als zentrale Begriffe vernetztes Vorgehen und Netzwerkarbeit, als deren entscheidende Instrumente für Forschung und Entwicklung. Ein wirkungsorientiertes, integriertes Management verbunden mit einer entsprechenden Personal- und Organisationsentwicklung sind für ihn entscheidend für weitere Innovationen sowohl im Strafvollzug und der Kriminalpolitik als auch in der Sozialwirtschaft. Aber alles das reicht nicht aus, wenn es nicht ethisch begründet und mit "politischem Unternehmertum« unterlegt ist, wie Andreas Tietze in einem schönen Beitrag zur Persönlichkeit des Jubilars zeigt.

Der Gewinn dieses Buches liegt darin, dass es an der Person Bernd Maelicke zeigt, welche arbeits- und fachübergreifende Dimensionen für innovative Entwicklungen in den verschiedenen Feldern der Sozialen Arbeit und der Sozialwirtschaft entscheidend sind und welche Bedeutung Wertschätzung für die Wertschöpfung in der Sozialwirtschaft hat.

Da das Buch, wie die Herausgeber betonen, nicht als Festschrift im traditionellen Sinne zu verstehen ist, sondern als Festgabe hätte es gerade deshalb auch einige kritische Stimmen vertragen, ohne dem unbestreitbaren Lebenswerk von Bernd Maelicke Abbruch zu tun. Insgesamt haben die Herausgeber aber ein interessantes und unterhaltendes Buch vorgelegt, das allen zu empfehlen ist, die die Entwicklung der Sozialwirtschaft und der Kriminalpolitik in Deutschland auch unter biografischen Gesichtspunkten interessiert.

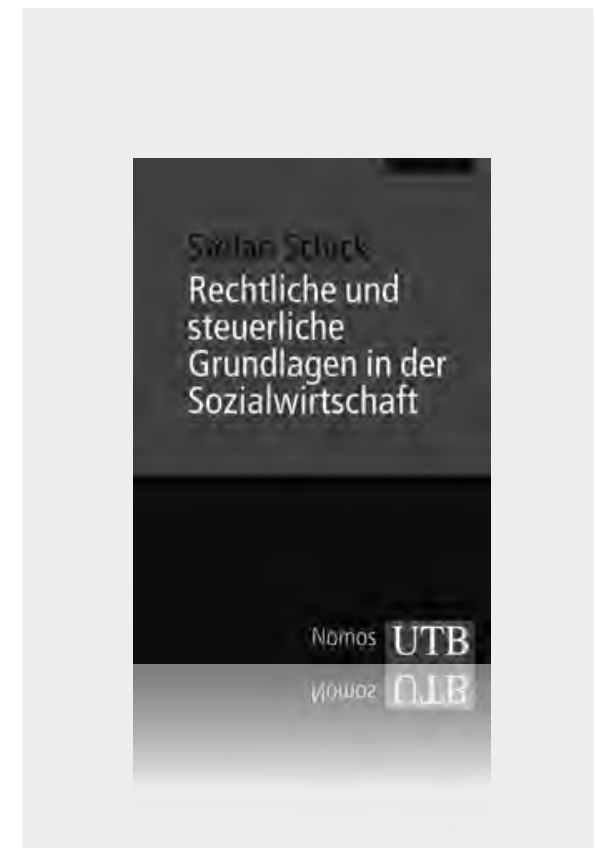

Rechtliche und steuerliche Grundlagen in der Sozialwirtschaft

Von RA Prof. Dr. Stefan Schick

2012, 211 S., brosch., 22,- $€$

ISBN 978-3-8252-3530-7

Erscheint ca. März 2012

Die Einführung erläutert die möglichen Rechtsformen sozialwirtschaftlicher Unternehmen, insbesondere der Rechtsform der $\mathrm{GmbH}$, des Vereins und der Stiftung und führt zudem systematisch in das Gemeinnützigkeits- und Steuerrecht ein, soweit dieses für sozialwirtschaftliche Einrichtungen von Bedeutung ist.

www.nomos-shop.de/13640 Nomos 